\title{
Time evolution of simple molecules during proto-star collapse
}

\author{
Ankan Das, ${ }^{\text {a }}$ Sandip K. Chakrabarti, ${ }^{\text {a b }}$ Kinsuk Acharyya ${ }^{\mathrm{b}}$ and Sonali Chakrabartia c \\ ${ }^{a}$ Centre For Space Physics, 43 Chalantika,Garia Station Road, Kolkata 700084, India, \\ email: ankan@csp.res.in

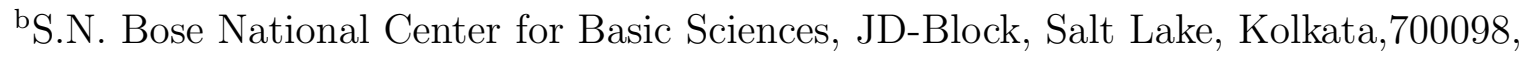 \\ India, email: sandip@csp.res.in, acharyya@csp.res.in, \\ 'Maharaja Manindra Chandra College, 20 Ramkanto Bose Street, Kolkata 700003,India, \\ email: sonali@csp.res.in
}

\section{Abstract}

We study the formation and evolution of several molecules in a collapsing interstellar cloud using a reasonably large reaction network containing more then four hundred atomic and molecular species. We employ a time dependent, spherically symmetric, hydrodynamics code to follow the hydrodynamic and chemical evolution of the collapsing cloud. The flow is assumed to be self-gravitating. We use two models to study the hydrodynamic evolution: in the first model, we inject matter into an initially low density 
region and in the second model, we start with a constant density cloud and let it collapse due to self-gravity. We study the evolution of the central core for both the cases. We include the grain chemistry to compute the formation of molecular hydrogen and carried out the effect of gas and grain chemistry at each time step. We follow the collapse for more than $10^{14}$ s (about 3 million years) and present the time evolution of the globally averaged abundances of various simple but biologically important molecules, such as glycine, alanine etc. We compare our results with those obtained from observations found that for lighter molecules the agreement is generally very good. For complex molecules we tend to under predict the abundances. This indicates that other pathways could be present to form these molecules or more accurate reaction rates were needed.

Keywords: hydrodynamics; star formation; ISM; chemical evolution

PACS No.: 95.30.Lz; 97.10. Bt;98.38.-j; 98.62.Bj

Details of the Corresponding Author:

Prof. Sandip K. Chakrabarti

S.N. Bose National Centre for Basic Sciences

JD Block, Salt Lake, Kolkata 700098, INDIA

Phone: +91 (033) 2335-5708, Fax : +91 (033) 2335-3477

e-mail: chakraba@bose.res.in, Mobile: +919903120700 


\section{Introduction}

More than 125 species of molecules have been observed in the interstellar clouds and star forming regions. Among them, over half are organic. Serious efforts have been made over the years to investigate the formation of such molecules in cool interstellar clouds in frigid conditions (Hasegawa et al., 1992; Hasegawa and Herbst, 1993; Leung et al., 1984; Prasad and Huntress, 1980a, 1980b). It is now quite certain that the most important building block, namely, the molecular hydrogen $\left(H_{2}\right)$ and some of the other lighter molecules must be produced in the presence of grains (Gould and Salpeter, 1963; Hollenbach and Salpeter, 1971; Hollenbach et al., 1971). Several analytical and numerical works have successfully shown how the molecular hydrogen may have been produced (Biham et al., 2001). A number of results are present in the literature where hydrodynamic and chemical evolutions have been attempted simultaneously. For example, Shalabiea and Greenberg (1995) used the pseudo as well as partially real time-dependent models for the hydrodynamical evolution. In the pseudo time-dependent method, they assumed a constant density and temperature of the cloud using which the chemical evolution was computed. In their time-dependent model, they included the density and temperature variations throughout the cloud. However, in their initial approach to the time-dependent modelling, they assumed a constant temperature but allowed only the density to vary. Ceccarelli et al. (1996) used the "inside-out", isothermal, spherical collapse model of Shu (1977) and coupled it with a time-dependent chemical evolution code. They included the heating and the cooling processes with an emphasis on the line emission. Shematovich et al. (1997) used Zeus 2D code which included the heating and the cooling. They present 
1D hydrodynamic and chemo-dynamical evolution of the proto-stellar cloud illuminated by the diffused interstellar UV radiation. They solved the equations of chemical kinetics, hydrodynamics and thermal balance simultaneously. In Lim et al. (1999) 2D numerical code was developed using the adaptive grid technique. Here, 454 reactions among 42 atomic and molecular chemical species were taken including the basic elements like $H$, $\mathrm{He}, \mathrm{C}, \mathrm{N}, \mathrm{O}$ and a representative low ionization potential metal $\mathrm{Na}$. At each grid point, the chemical evolution was followed by a calculation of the reaction rates using the local conditions obtained from the hydrodynamical flow. They primarily concentrated on the diffused clouds and emphasized the interfaces of the interstellar media and resulting dynamical mixing. Aikawa et al. (2005) studied time-dependent evolution of BonnerEbert spheres by assuming clouds having a specific parameter $\alpha$ which is the ratio of the gravitational force to the pressure force. Recently, Acharyya et al. (2005) solved the Master equations and rate equations of Biham et al. (2001) for various cloud parameters and followed the evolution of $H_{2}$ as a function of time. Both this work and the earlier works of Chakrabarti and Chakrabarti (2000a) employed steady state matter distribution and assumed that the density and the temperature distributions at a given radial distance do not change with time. Chakrabarti and Chakrabarti (2000a) used a large number of species and the reaction rates were taken from the UMIST data base. Some of the reaction rates which were not available in the literature were assumed to be similar to other two body reactions. Subsequently, these new and assumed reaction rates were parametrized (with reaction rates up to a thousand times smaller compared to Chakrabarti and Chakrabarti (2000a) to include the effect of the size of the reactant molecules (Chakrabarti and Chakrabarti, 2000b). It was shown that even under frigid 
and tenuous conditions of the interstellar media, a significant and perhaps a detectable amount of simple amino acids and even important ingredients of DNA molecule (such as adenine) may form. Ceccarelli et al. (2000) estimated the upper limit of the abundance of glycine to be about $10^{-10}$ (cooler outer cloud) to $7 \times 10^{-9}$ (hot core). Kuan et al. (2003) estimated the fractional abundance of glycine to be $2.1 \times 10^{-10}$ for Sgr B2, $1.5 \times 10^{-9}$ for Orion, and $2.1 \times 10^{-10}$ for W51. These numbers are comparable to what was predicted in Chakrabarti and Chakrabarti (2000a), however, there are clearly some debate on the possible pathways for the formation of glycine with the route followed in the Chakrabarti and Chakrabarti (2000a, 2000b). Similarly, there are also some debate on whether glycine is actually observed (Hollis et al., 2003; Snyder et al., 2005). A few other relevant results which may be mentioned in passing are as follows. Tarafdar et al. (1985) presented a model of chemical and dynamical evolution of isolated, initially diffused and quiescent interstellar clouds. A semi-empirically derived dependence of the observed cloud temperatures on the visual extinction and density was used in this work. Sorrell (2001) outlined a theoretical model for the formation of the interstellar amino acids and sugars. In this model, first ultraviolet photolysis creates a high concentration of free radicals in the mantles and the heat input due to the grain-grain collision causes radicals to react chemically with another to build complex organic molecules. Bernstein et al. (2002) reported a laboratory demonstration that the complex bio-molecules like glycine, alanine etc. are naturally formed from the ultraviolet photolysis of the interstellar grains. Munoz Caro et al. (2002) report the detection of amino acids even at room temperature on an interstellar ice analogue that was irradiated with ultraviolet light in a high vacuum at $12 \mathrm{~K}$. Altogether sixteen amino acids were identified. The results demonstrate that a 
spontaneous generation of amino acids in the interstellar medium is possible, supporting the suggestion that prebiotic molecules could have been delivered to the early earth by cometary dust, meteorites or interplanetary dust particles.

In this backdrop of this observational and experimental status, we carry out our investigation by improving earlier work by first incorporating the accurate grain chemistry as elaborated in Acharyya et al. (2005) and then by actually combining the results of a time dependent hydrodynamics code with the chemical evolution code to see how the abundances vary with the grid locations. We also chose initial conditions very much different from the earlier studies. We used two different but realistic models. In one model (Model A), the cloud matter is injected through the grid boundary at a constant speed and the cloud as well as the core are allowed to form ab initio. In the other model (Model B), the computational grid area is chosen to be the central part of a much larger spherical cloud of constant density and temperature. The rate at which the interstellar matter enters into the grid (i.e., the rate at which the larger cloud is evacuated) depends on the gravitational pull between the inner cloud within computatioal grid and the outer cloud outside of the computational grid. In following the chemical evolution, we used the available standard reaction rates for most of the reactions, but the rates of some of the very complex molecule formation are still very much uncertain and as such we present the evolution of the mass fractions only for simpler bio-molecules. In the next section, we present the hydrodynamic equations which govern the cloud collapse. In $\S 3$, we present the hydrodynamical and chemical evolution. In $\S 4$, we discuss in detail the nature of the cloud models which we simulate and how the chemical evolution code is used in conjunction with the results of the hydrodynamic simulation. In $\S 5$, we present the results. Finally, in $\S 6$, we make 
concluding remarks.

\section{Hydrodynamic Equations Governing the Cloud Collapse}

The time dependent simulations have been carried out by various authors using both the Lagrangian and Eulerian methods. For instance, Prasad et al. (1991) and Tarafdar et al. (1985) used a Lagrangian scheme with a semi-empirical approach for the energy equation. Shalabiea and Greenberg (1995) used a partially time dependent model in which only the densities are allowed to be time dependent during collapse of the cloud. We have used a finite difference Eulerian scheme (upwind scheme) in which we difference the one dimensional Euler equations along the radial grid. We consider a self-gravitating, collapsing, spherically symmetric gas cloud. We choose the co-ordinate system to be $(r, \theta, \phi)$ with origin at the center of the proto-star. Since we would be interested in the spherical case, we ignore the $\theta$ and $\phi$ component motions and concentrate only on the radial equation in this paper. The Eulerian equations of hydrodynamics written in spherical coordinates are given by:

$$
\frac{\partial \rho}{\partial t}+\frac{1}{r^{2}} \frac{\partial\left(\rho v_{r} r^{2}\right)}{\partial r}=0
$$

and

$$
\frac{\partial \rho v_{r}}{\partial t}+\frac{1}{r^{2}} \frac{\partial\left(\rho v_{r}^{2} r^{2}\right)}{\partial r}=-\left(\rho \frac{\partial \Phi}{\partial r}+\frac{\partial p}{\partial r}\right)
$$

Here, $\Phi$ is the gravitational potential $[=-G M(r) / r]$ where, $M(r)$ is the mass of the cloud inside radial distance $r$. 
We assume the ideal gas equation to be,

$$
p=\rho k T / \mu m_{p}
$$

where, $k$ is the Boltzmann constant, $T$ is the local temperature, $\mu$ is the mean molecular weight, $m_{p}$ is the proton mass.

\section{Hydrodynamical and Chemical Evolution}

The equations presented in the previous Section have been solved to study full timedependent behaviour of the spherical flow. The actual solution depends on the model flow. In the present paper we used two types of initial conditions for the evolution of two hydrodynamic models. These are coupled with chemical evolution code to obtain time dependent chemical composition.

\subsection{Hydrodynamical Models}

(a) Model A: Here we start with a grid of size $r_{\text {out }}$. We assume that initially the cloud contains a negligible amount of mass. At the outer boundary, we inject matter at a constant rate of $\frac{d M}{d t}=4 \pi \rho_{\text {out }} v_{\text {out }} r_{\text {out }}^{2}$, where, $\rho_{\text {out }}$ is the injection density and $v_{\text {out }}$ is the injection velocity at the outer boundary $\left(r=r_{\text {out }}\right)$. Using this model, we mimic the formation of the cloud itself from a supply of matter from a large reservoir of diffused gas.

The inner boundary is chosen at $r=r_{i n}$. The distance $r_{\text {out }}-r_{\text {in }}$ is divided into $N=100$ logarithmically equal spaced grid points. At each time step, the mass of the core is dynamically updated by the amount of matter that is getting inside $r_{i n}$. The rate 
of increase of $M_{\text {core }}$ is,

$$
\frac{d M_{\text {core }}}{d t}=4 \pi \rho_{\text {in }} v_{\text {rin }} r_{i n}^{2}
$$

where, $\rho_{i n}$ is the density at the inner grid point, $v_{\text {rin }}$ is the velocity at the inner grid point, $r_{i n}$ is the radial distance of the inner grid point from the center of the molecular cloud. Similarly, the mass of the cloud is also dynamically updated. The flow is assumed to be isothermal throughout and $T=10 \mathrm{~K}$ is assumed for concreteness.

(b) Model B: In this model, we assume that we have a finite sized $r=r_{r e s}$ reservoir of matter and we are considering only the inner region of size $r_{\text {out }}$ for computational purpose. The matter is coming from the reservoir and it is evacuated to form the star itself. The inward velocity is computed self-consistently from the gravitational pull between the matter inside $r_{\text {out }}$ and that outside of $r_{\text {out }}$. The whole cloud is assumed to be isothermal (with $T=10 \mathrm{~K}$ ) and has a constant density $\rho_{\text {int }}$ throughout. The size of the whole cloud is $r_{\text {res }}>>r_{\text {out }}$. We assume that $M_{\text {total }}=M_{\text {core }}+M_{\text {cloud }}+M_{\text {out }}$, where, $M_{\text {core }}$ is the core mass within $r=r_{i n}$ which will increase at the same rate as in eq. (4), $M_{\text {cloud }}$ is the mass of the cloud within the computational grid, i.e., in between $r_{i n}$ and $r_{\text {out }}$ and $M_{\text {out }}$ is the mass in between the radius $r_{\text {res }}$ and $r_{\text {out }}$. Thus, $M_{\text {out }}$ is the reservoir mass which is depleted as matter is injected within $r=r_{\text {out }}$. The injection velocity is dynamically calculated from the following way. First, we note that an approximate expression for the acceleration of matter at the outer edge of the computational grid could be taken as,

$$
f_{\text {out }} \sim \frac{G\left(M_{\text {cloud }}+M_{\text {core }}\right)}{r_{m}^{2}}
$$


where, $r_{m}=\left(r_{r e s}+r_{\text {out }}\right) / 2$. Hence the injection velocity is,

$$
v_{\text {out }} \sim \sqrt{f_{\text {out }} r_{m}},
$$

and the average density of the injected matter is,

$$
\rho_{\text {out }}=\frac{M_{\text {out }}}{V_{\text {out }}},
$$

where, $V_{\text {out }}=4 / 3 \pi\left(r_{\text {res }}^{3}-r_{\text {out }}^{3}\right)$. By this process, the large cloud would be gradually evacuated as the star is formed. The cloud mass $M_{\text {cloud }}$ inside $r_{\text {in }}<r<r_{\text {out }}$ will increase at the rate,

$$
\frac{d M_{\text {cloud }}}{d t}=4 \pi\left(\rho_{\text {out }} v_{\text {out }} r_{\text {out }}^{2}-\rho_{\text {in }} v_{\text {in }} r_{\text {in }}^{2}\right)
$$

where, $v_{i n}$ and $\rho_{i n}$ are respectively the velocity and density at the inner boundary $r_{i n}$.

\subsection{Chemical Evolution}

The chemical evolution code is similar to that used in Chakrabarti and Chakrabarti (2000a, 2000b) except in one very important way, namely, the way we handle the grain chemistry. In Chakrabarti and Chakrabarti (2000a, 2000b) a constant rate of recombination of $H+H \rightarrow H_{2}$ was chosen following the prescription of Miller et al. (1997). In our present work, however, we incorporate the grain chemistry properly for the production of $H_{2}$ at each radius. Here, we followed the techniques used in Acharyya et al. (2005). We used only the olivine grains for simplicity. We also incorporate the grain size distribution according to Weingartner and Draine (2001a, 2001b) and sub-divided the grains into three major types (a) $5 \AA$, (b) $75 \AA$, and (c) $0.2 \mu$ so that the calculation of $H_{2}$ becomes faster. 
Whether or not the Master equation or the rate equation would be used depends on the flux of the infalling hydrogen (Acharyya et al., 2005) on the grain surface. We take the reaction rates from Miller et al. (1997) which supplies the rate constant $k$ for a two body reaction as:

$$
k=\alpha(T / 300)^{\beta} \exp (-\gamma / T) \mathrm{cm}^{3} \mathrm{~s}^{-1},
$$

where, $\alpha, \beta, \gamma$ are constants. The reactions for which the rates were not available in the UMIST data base, we take a conservative value of $\alpha=10^{-10} \mathrm{~cm}^{-3} \mathrm{~s}^{-1}, \beta=\gamma=0$ like any other typical two body (open shell) reactions even though we may be using neutral-neutral reactions. The chemistry of bio-molecule formation in space is very much unknown and it is difficult to quantify the rate with any certainty. Thus the results obtained using these rates may have some errors.

Two models have been run with different initial abundances of carbon. Generally, we keep the initial composition as was kept in Miller et al. (1997) (after converting to mass fractions as in Chakrabarti and Chakrabarti, 2000a), i.e., H:He:C:N:O:Na:Mg:Si:P:S:Cl:Fe $=0.64: 0.35897: 5.6 \times 10^{-4}: 1.9 \times 10^{-4}: 1.81 \times 10^{-3}: 2.96 \times 10^{-8}: 4.63 \times 10^{-8}: 5.4 \times 10^{-8}: 5.79 \times$ $10^{-8}: 4.12 \times 9^{-7}: 9 \times 10^{-8}: 1.08 \times 10^{-8}$. This Set is our 'Set 1' initial abundance. In 'Set 2' initial abundance, we used a higher amount of carbon to simulate the chemical evolution inside a collapsing cloud in the environment of evolved stars.

While we include all the reactions given in the UMIST data base, we wish to remind that since the interstellar medium is cooler, the exothermic reactions should be more favourable. The following major types of the reactions are included (Tielens, 2005):

\section{(i) Photochemistry:}


The UV photons present in the diffuse ISM are a dominant destruction agent for small molecules. Inside a cloud, the radiation field will be attenuated by the dust. In the unshielded radiation field this type of reactions have a typical rate of $10^{-9} \mathrm{~s}^{-1}$. These reactions mainly occur in the outer edge of the molecular cloud where UV photons are profuse in number. Deep inside the cloud some UV photons can be created due to radiative association, which could also lead to this type of reactions. The rate is calculated by,

$$
\kappa_{p d}=\operatorname{aexp}\left[-b A_{v}\right]
$$

where, $A_{v}$ is the visual extinction due to the dust, $a$ is the unshielded rate and $b$ is the self-shielded rate. An example of this kind of reaction is,

$$
C H+h \nu \rightarrow C+H
$$

(ii) Neutral-neutral: This type of reactions often possesses appreciable activation barriers because of the necessary bond breaking associated with the molecular re-arrangement. This type of reactions is important when the gas is warm, e.g., in stellar ejecta, in hot cores associated with proto-stars, in dense photo-dissociation regions associated with luminous stars, or in the post-shock regions. The rates are generally around $4 \times 10^{-11} \mathrm{~cm}^{3} \mathrm{~s}^{-1}$ when no activation barrier is present. Reaction rates are calculated as,

$$
\kappa=\alpha(T / 300)^{\beta} \exp (-\gamma / K T)
$$

where, $\alpha, \beta, \gamma$ are used to calculate the rate coefficients. One example of this type of 
reaction is,

$$
H_{2}+\mathrm{O} \rightarrow \mathrm{OH}+\mathrm{H}
$$

In general, the only neutral-neutral reactions that occur in the cold conditions of the dark clouds are those involving atoms or radicals, often with non-singlet electronic ground states that do not have activation barriers. One example of this type of reaction is,

$$
\mathrm{C}+\mathrm{OH} \rightarrow \mathrm{CO}+\mathrm{H}
$$

(iii) Ion-molecule reactions: Exothermic ion-molecule reactions occur rapidly because the strong polarization-induced interaction potential can be used to overcome any activation barrier energy involved. In the exothermic direction, this kind of reactions have a typical rate of $2 \times 10^{-9} \mathrm{~cm}^{3} \mathrm{~s}^{-1}$ and the reaction rates are in the form

$$
\kappa=\alpha
$$

One example of this kind of reaction is,

$$
H_{2}^{+}+H_{2} \rightarrow H_{3}^{+}+H
$$

(iv) Charge transfer reactions: This type of reactions is of great importance for setting the ionization balance in the HII region. The charge exchange between $\mathrm{O}$ and $\mathrm{H}^{+}$ is a very important reaction in the ISM because it ionizes the oxygen which is then able to participate in the chemistry of the ISM. This type of reactions has a typical rate of 
$10^{-9} \mathrm{~cm}^{3} \mathrm{~s}^{-1}$ in the exothermic direction. An example is:

$$
\mathrm{H}^{+}+\mathrm{O} \rightarrow \mathrm{H}+\mathrm{O}^{+}
$$

(v) Radiative association reactions: In this type of reaction, the product after the collision of two species is stabilized through the emission of a photon. The reactions of this type have highly reaction specific rates. The reaction rates are given by,

$$
\kappa=\alpha .
$$

One example of this type of reaction is,

$$
H+C \rightarrow C H+h \nu
$$

(vi) Dissociative electron recombination reactions: This type of reactions involves in the capture of an electron by an ion to form a neutral in an excited electronic state that can dissociate. Typical rate of such a reaction is around $10^{-7} \mathrm{~cm}^{3} \mathrm{~s}^{-1}$ and the rate coefficient is calculated by,

$$
\kappa=\alpha(T / 300)^{\beta} \text {. }
$$

One example of this kind of reaction is,

$$
O H^{+}+e \rightarrow O+H
$$

(vii) Associative detachment reactions: In this case, an anion and an atom collide 
and the neutral product stabilizes through an electron emission. This type of reactions has a rate of around $10^{-9} \mathrm{~cm}^{3} \mathrm{~s}^{-1}$ in the exothermic direction.

$$
H+H^{-} \rightarrow H_{2}+e
$$

(viii) Collisional association: In laboratory settings, three-body reactions generally dominates chemistry,

$$
A+B+M \rightarrow A B+M
$$

with rates $\approx 10^{-32} \mathrm{~cm}^{6} \mathrm{~s}^{-1}$. These reactions generally have very little importance in the astrophysical environment except for dense gas near stellar photosphere or in dense $(\approx$ $10^{11} \mathrm{~cm}^{-3}$ ) circumstellar disks.

We do not give the reaction pathways since there would be too many of them. Generally, pathways given in Chakrabarti and Chakrabarti (2000a, 2000b) have been used for synthesis of those complex molecules which are absent in the UMIST data base.

\section{Results}

Armed with a working time-dependent hydrodynamical evolution code and a chemical evolution code, we ran two models (Models A and B) of the interstellar cloud collapse. In both the Models we choose, $r_{i n}=3.98 \times 10^{13} \mathrm{~cm}$ and use 100 grid points which are spaced equally in the logarithmic scale along the radial direction. We assume that anything going inside $r_{i n}$ will increase the mass of core while the the radius of the core remains $r_{\text {in }}$. In Model A, we chose $r_{\text {out }}=3.54 \times 10^{18} \mathrm{~cm}$ and we ran for both the Sets $1-2$ as the initial compositions. We started our simulation with $\rho_{i n t}=10^{-27} \mathrm{gm} \mathrm{cm}^{-3}$ in all the grids 
(except on the boundary, see below). Thus, we start with a core of a negligible mass of $(4 / 3) \pi r_{i n}^{3} \rho_{i n t}=2.64 \times 10^{14} \mathrm{gm}$. We inject matter at $v_{i n t}=50 \mathrm{~cm} \mathrm{~s}^{-1}$ at each grid point in the outer boundary.

In Model B1, we combine the hydrodynamical code and the chemical evolution code using only the Set 1 as the initial composition. Here, we choose $r_{\text {res }}=10^{20} \mathrm{~cm}, r_{\text {out }}=$ $3.16 \times 10^{18} \mathrm{~cm}$ and $r_{i n}=3.98 \times 10^{13} \mathrm{~cm}$ and use equal logarithmic radial spacings as before. At the start of the simulation $\rho_{\text {int }}=10^{-22} \mathrm{gm} \mathrm{cm}^{-3}$ and $v_{\text {int }}=10 \mathrm{~cm} \mathrm{~s}^{-1}$ at each grid point. Initially, the core mass was $(4 / 3) \pi r_{i n}^{3} \rho_{i n t}=2.64 \times 10^{19} \mathrm{gm}$ in this Model. In Model B2, we choose $r_{\text {res }}=3.16 \times 10^{18} \mathrm{~cm}, r_{\text {out }}=3.16 \times 10^{17} \mathrm{~cm}$ and other conditions are same as above.

As far as the chemical evolution is concerned, we use a large network of about 4000 reactions and solve 422 equations simultaneously each of which is to update the mass fraction of one single specie. However, in order to simplify the chemical evolution and to save the computational time, we do not run the chemical evolution code at each grid at each time step. Instead, we divide the entire region under consideration in ten logarithmically equal spaced zones along the radial direction. Appropriately weighted averaged density is used in each such zone. The initial abundance of matter in each zone is updated using the existing abundance in that zone plus the abundance of the upstream matter advecting in and minus the abundance of the downstream matter advecting out. The time step in chemical evolution is totally dictated by the fastest reaction in the network. The evolution process is continued till the end of the hydrodynamic simulation. While we have time evolution of the abundance of each species at each zone, we plot only the global average of the abundances just to give an idea of how the average abundance is 
evolving.

\subsection{Results of Models A and B with Set 1 as initial composition}

In this Model, we choose $\rho_{\text {out }}=10^{-22} \mathrm{gm} \mathrm{cm}^{3}, v_{\text {out }}=10^{4} \mathrm{~cm} \mathrm{~s}^{-1}$. Thus, the rate of injected matter is given by $\dot{M}=4 \pi \rho_{\text {out }} v_{\text {out }} r_{\text {out }}^{2}=1.57 \times 10^{20} \mathrm{gm} \mathrm{s}^{-1}$. This matter moves in due to self-gravity of the cloud and the attraction of the core. The simulation was carried out till a few times $10^{6}$ yr. Figure 1a shows how the velocity profile of the flow changes with time. Time (in years) is marked on each curve. A flow which began with a constant velocity, eventually assumes an almost steady state $\left(v(r) \sim r^{-\alpha}\right.$ with $\left.\alpha \sim 4 / 5\right)$. The evolution of the density distribution is shown in Figure 1b. Here too, an initially constant density distribution assumes a power-law distribution of $\rho(r) \sim r^{-3 / 2}$ toward the end of the simulation. Time (in years) is marked on each curve. Note that once the core becomes massive, it starts to evacuate the grids and thus the density over the entire cloud gradually decreases as is shown by the dot-dashed curve drawn at $\sim 6.33 \times 10^{6} \mathrm{yr}$. After about $t=10^{13} \mathrm{~s}$, i.e., about $3 \times 10^{5} \mathrm{yr}$, densities on each grid started increasing after the empty grids are filled in by the inflowing matter. This is shown in Figure 1c where the time evolutions of the densities on the 50th grid (solid curve) and the innermost grid (dotted curve) are shown. Towards the end, we note that the densities at these grids gradually decrease as the cloud starts to become empty. Meanwhile, the core starts to grow because of the mass accretion during this period. Figure $1 \mathrm{~d}$ shows how the mass of the core is evolving.

In Model B1, the velocity assumes almost steady state after some initial transient time as before. The evolution of velocity is shown in Figure 2a. In Figure 2b, the evolution of 

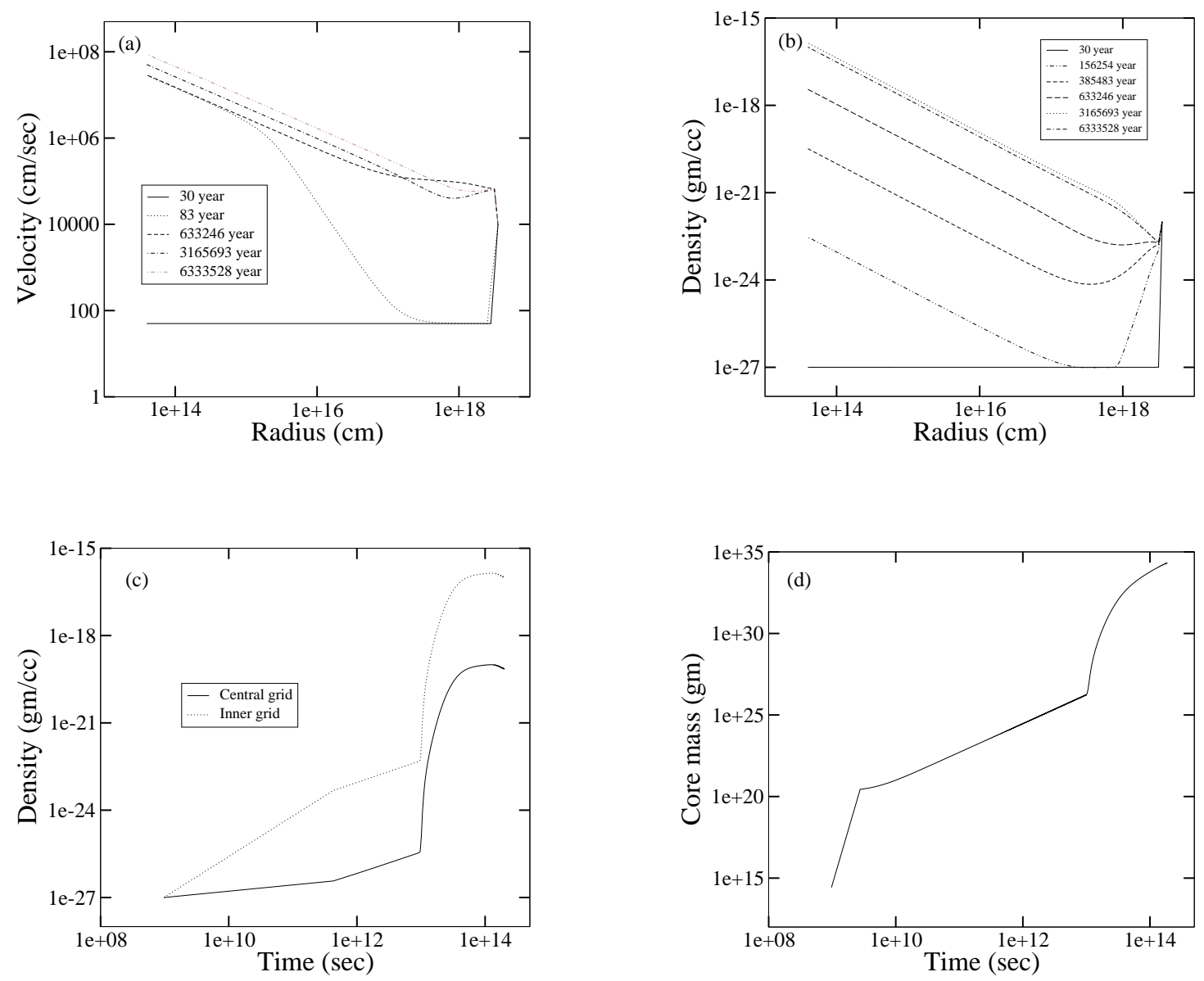

Figure 1. Evolutions of (a) the velocity and (b) the density distribution inside the collapsing cloud of a Model A simulation. Also shown are the evolutions of the density at (c) the middle (solid) grid and the innermost grid (dotted) and (d) the growth of the proto-stellar core with time. The densities in the grid have a sharp rise after the initially empty grid is filled in by the infalling matter at about $10^{13} \mathrm{~s}$. From this time onward, the core also starts growing rapidly. 

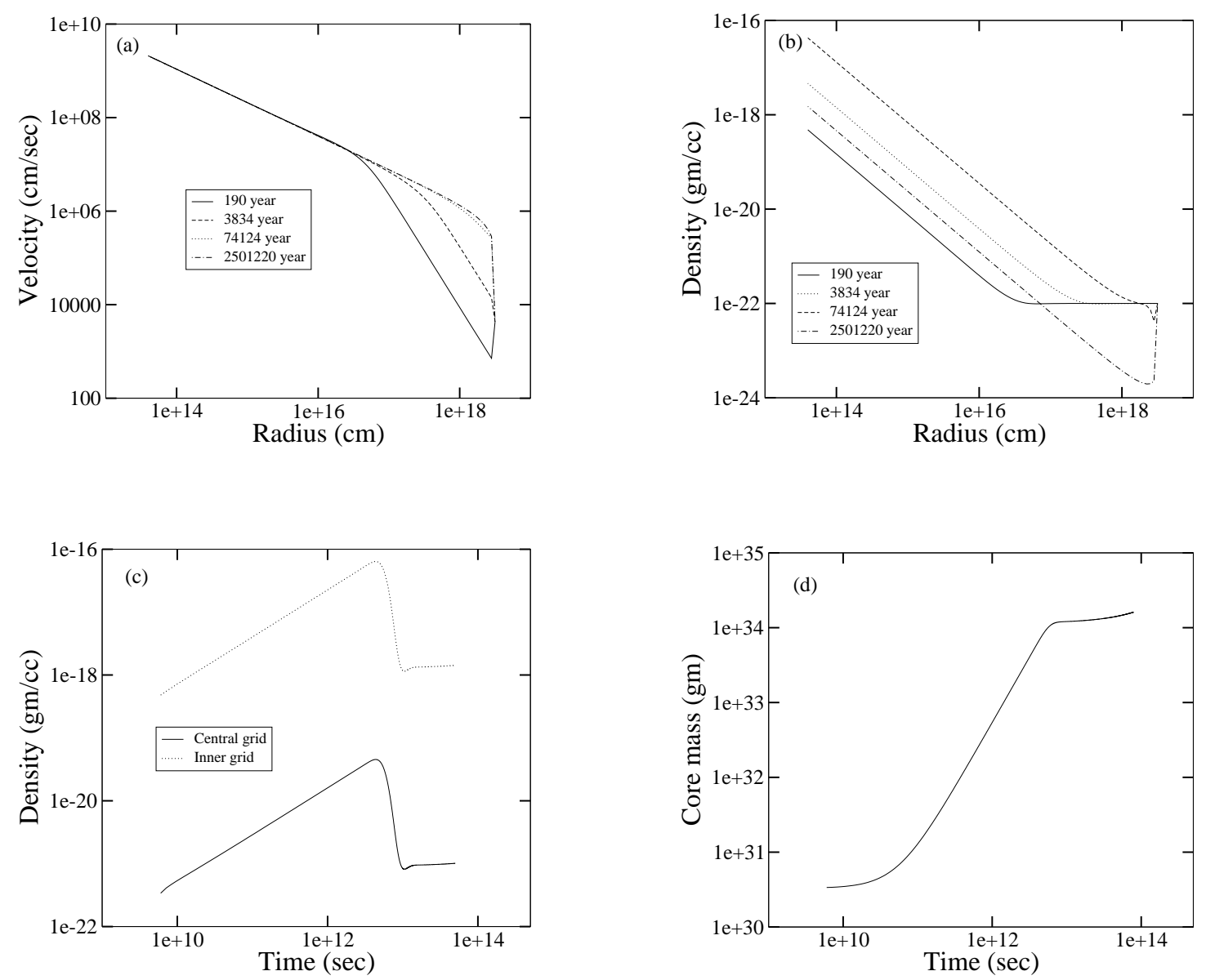

Figure 2. Same as Figs.1(a-d) except that Model B1 is used for simulation. In this case, the depletion of matter from a cloud of fixed mass takes place and the core mass of the cloud goes up initially rapidly and slowly afterwards. 
density is shown. Initially, the density of the cloud increases and new matter is injected from the cloud external to the grid, but as the time proceeds, the core grows while the external cloud is evacuated. This results in eventual decrease in density of the cloud as shown by the dot-dashed curve in Figure 2b. Similarly, from Figure 2c, it is clear that the density at the middle grid (solid curve) and the innermost grid (dotted curve) will also go down due to the growth of the core mass. In Figure $2 \mathrm{~d}$, the evolution of core is shown. Unlike in the previous model, the core mass starts growing from the very beginning of the simulation, but the rate of growth is slowed down when the cloud mass is depleted.

Our Model A and Model B are two complimentary models. In Model A, the initial grid was almost empty and thus the core mass was growing slowly. Eventually the matter injected at the our boundary caught up and the core grew rapidly. In Model B, the initial grid was full. After it is evacuated, matter from the reservoir is accreted slowly. Thus, initially, the core grows rapidly but the rate of growth slowed down in the late phase.

We now show the variation of different species for Model A hydrodynamic simulation. Figures $3(\mathrm{a}-\mathrm{d})$ show the time variation of the average abundances of several species using the initial composition as in Miller et al. (1997) (Set 1). In Figure 3a we show the evolution of $\mathrm{H}$ and $\mathrm{H}_{2}$. The mass fraction of the atomic hydrogen goes down since it is utilized to form $\mathrm{H}_{2}$ and other hydrogenated species. Since the atomic hydrogen is very much reactive agent it goes to the molecular form easily and thus towards the end of our simulation almost all the $H$ was found primarily in the form of $H_{2}$. In Figure $3 \mathrm{~b}$, we show the abundances of $\mathrm{O}_{2}, \mathrm{H}_{2} \mathrm{O}$ and $\mathrm{CO}$ whose final mean mass fractions are around $10^{-5}, 10^{-7}$ and $10^{-3}$ respectively. The production rate suddenly increases after the grids are filled up with the collapsing shell of matter and the densities go up. In Figure 3c, 

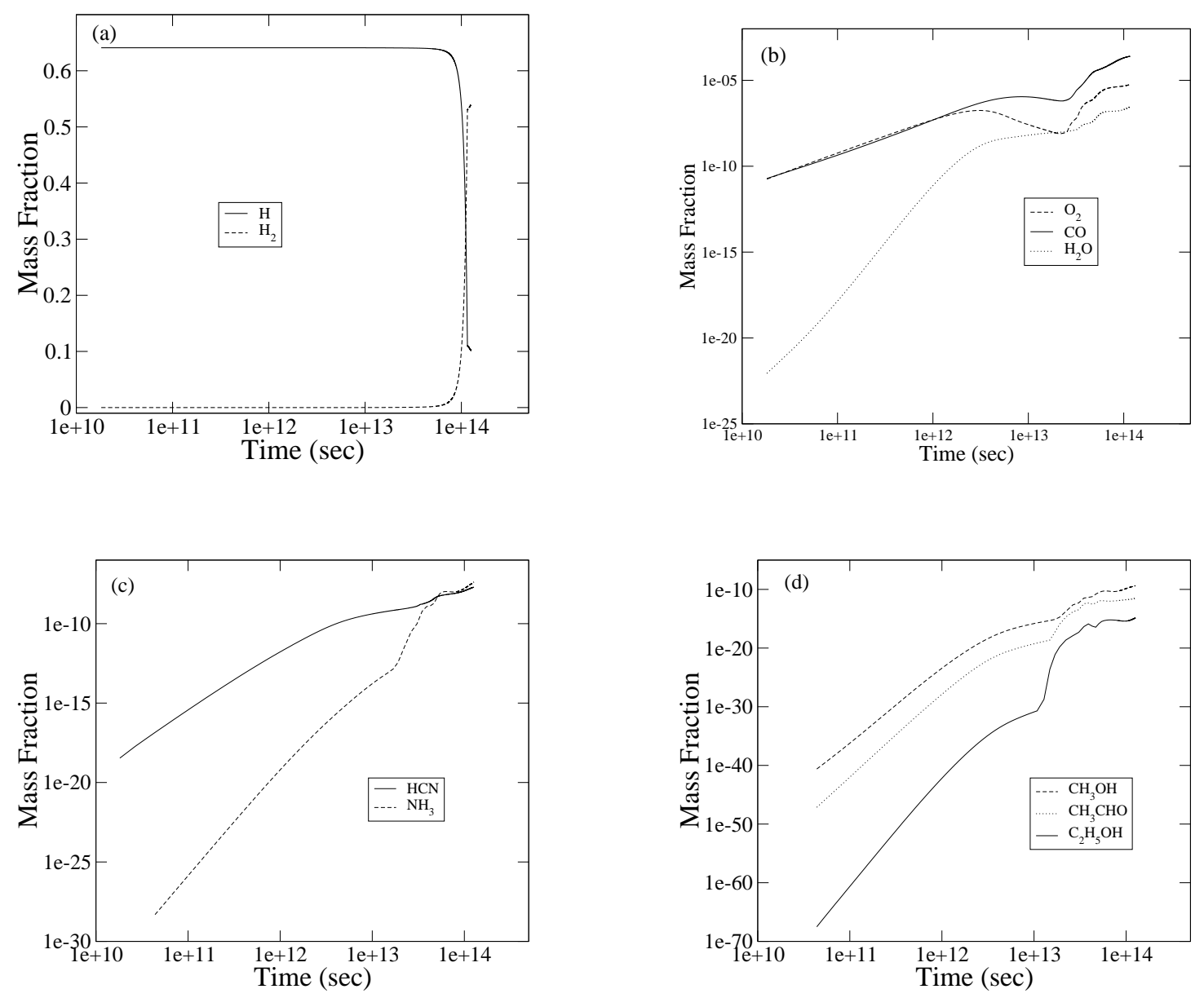

Figure 3. Time variation of the spatially averaged mass fractions of (a) $\mathrm{H}$ and $\mathrm{H}_{2}$, (b) $\mathrm{O}_{2}, \mathrm{H}_{2} \mathrm{O}$ and $\mathrm{CO}$ (c) $\mathrm{HCN}$ and $\mathrm{NH}_{3}$ and (d) $\mathrm{C}_{2} \mathrm{H}_{5} \mathrm{OH}, \mathrm{CH}_{3} \mathrm{CHO}$ and $\mathrm{CH}_{3} \mathrm{OH}$. Here, Model A cloud is chosen and the initial mass fractions are the same as that of Set 1. 


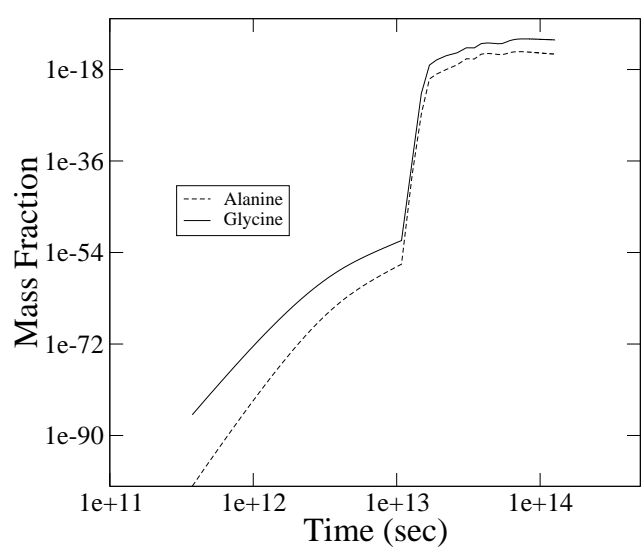

Figure 4. Time variation of the spatially averaged mass fractions of two simple biomolecules, such as glycine and alanine. The hydrodynamic and chemical model are the same as in the previous Figure.

the time dependences of the abundances of $\mathrm{HCN}$ and $\mathrm{NH}_{3}$ are shown. The final mass fractions of these species are around $\sim 10^{-7}$. In Figure $3 \mathrm{~d}$, we show the variations of $\mathrm{C}_{2} \mathrm{H}_{5} \mathrm{OH}, \mathrm{CH}_{3} \mathrm{CHO}$ and $\mathrm{CH}_{3} \mathrm{OH}$ whose final mass fractions are $\sim 10^{-15}, \sim 10^{-10}$ and $\sim 10^{-9}$ respectively. Finally, in Figure 4, we show the evolutions of some of the simple bio-molecules, such as glycine and alanine whose final mass fractions are found to be around $\sim 10^{-13}$ to $\sim 10^{-15}$ respectively.

\subsection{Collapse of clouds with other initial carbon abundances and comparison with observations}

Since the initial composition of the clouds is not known very accurately, we ran Model A for one more initial chemical abundance (Set 2), where we use the mass fraction of carbon to be 0.001 , roughly twice as much as we used in Set 1 above. This may mimic the environment of an evolved star. We adjusted the mass fraction of helium accordingly so as to keep the sum of the mass fractions to be unity. As expected, the carbon containing 


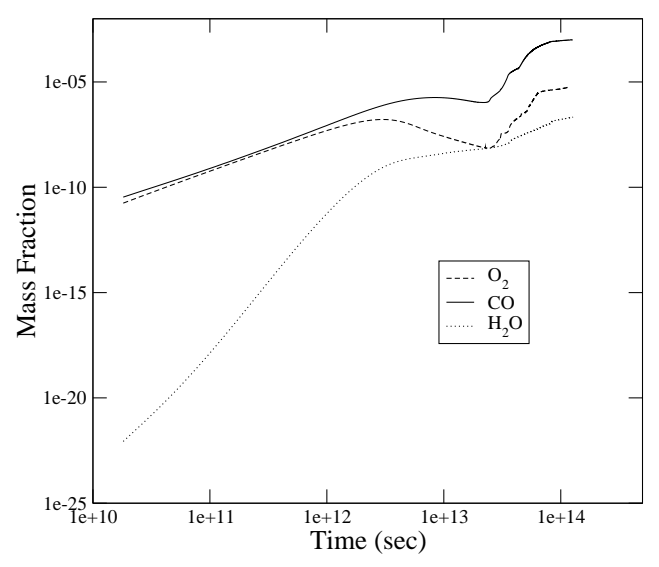

Figure 5. Time variation of the spatially averaged mass fractions of $\mathrm{H}_{2} \mathrm{O}$ and $\mathrm{CO}$ when the initial carbon abundance is twice that in Set 1.
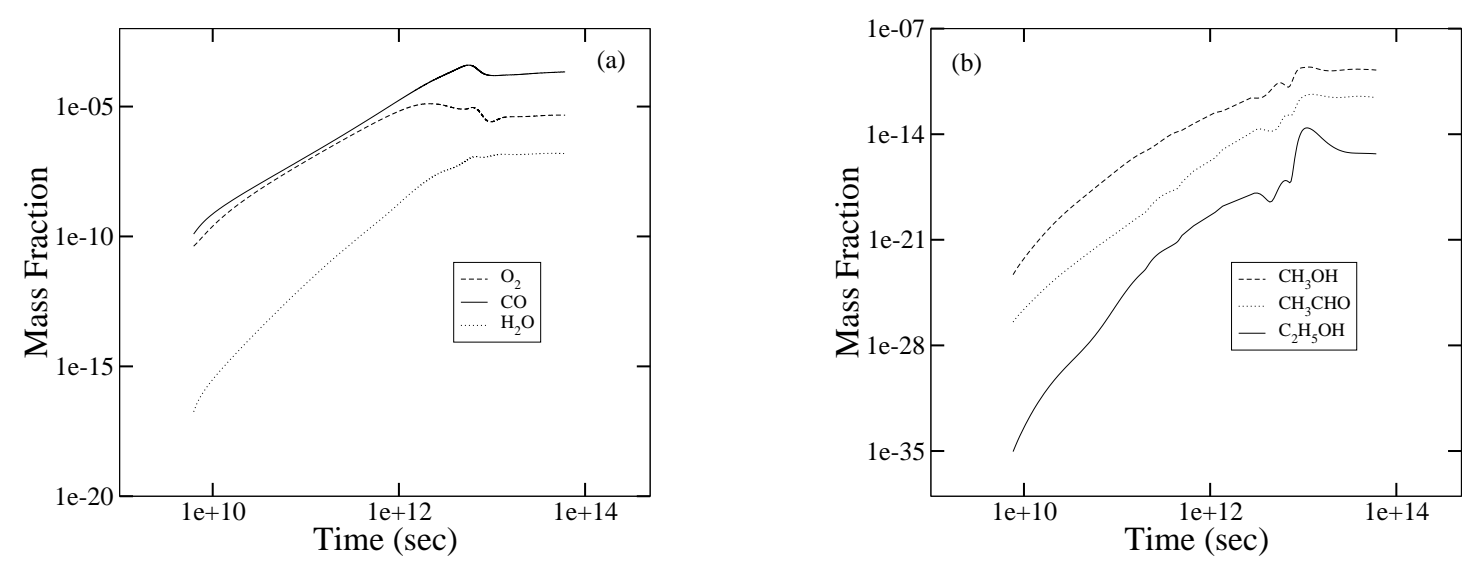

Figure 6. Time variation of the spatially averaged mass fractions of (a) $\mathrm{H}_{2} \mathrm{O}$ and $\mathrm{CO}$ (b) $\mathrm{C}_{2} \mathrm{H}_{5} \mathrm{OH}, \mathrm{CH}_{3} \mathrm{CHO}$ and $\mathrm{CH}_{3} \mathrm{OH}$. Here Model B1 was chosen.

species become more abundant in simulations with Set 2 as the initial abundance. In Figure 5, we present the variations of $\mathrm{O}_{2}, \mathrm{H}_{2} \mathrm{O}$ and $\mathrm{CO}$ with time for these two models. These are to be compared with those presented in Figure 3b.

Figures $6(\mathrm{a}-\mathrm{b})$ show the time variation of the average abundances of several species using the initial composition of Model B1. In this connection, we wish to note that though we have been using the so-called freeze-out of the chemical species, the effect is 

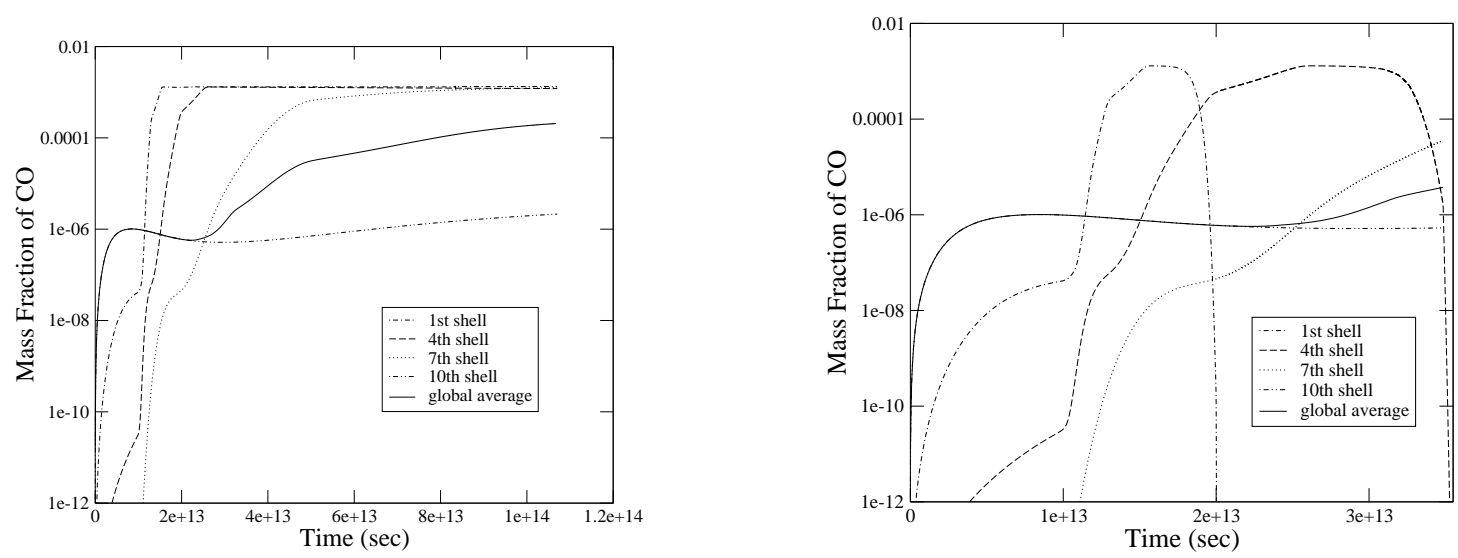

Figure 7. Evolution of the mass fraction of CO (a) without and (b) with the freeze-out effect taken into account. The results in the 1st, 4th, 7th and 10th shells are displayed. Note that in the 1st and the 4th shell, the freeze-out effect is prominent. However, the global averages in both the cases do not differ by any large margin since they are dominated by the outer shells. Evolution in (b) only up to the freeze-out time is depicted to show detailed variation.

not very easy to see in our abundance plots. This is because we are plotting the globally averaged abundances which is dominated by very slowly evolving matter in the outermost cell. By the time the matter is dense and more evolved, it is compressed to a smaller volume and does not contribute much in the weighted average. However, when we plot the evolution for each cell, we see that the freeze-out effect can be seen in the innermost four cells. In Figures $7(\mathrm{a}-\mathrm{b})$ we show the evolutions of the mass fraction of $\mathrm{CO}$ in the 1st (innermost), 4th, 7th and 10th (outermost) shells of matter in the cloud when (a) we do not consider the freeze-out effect and when (b) we do consider the freeze-out effect. When no freeze-out is considered (Figure 7a), all the shells eventually reach a saturation value of the mass-fraction. This is reached when the enhancement of the CO through combination of $\mathrm{C}$ and $\mathrm{O}$ matches its reduction through hydrogenation. Different shells take different times depending on the average density of each shell. When we assume a 
Table 1

Comparison of our Results with the observed abundances

\begin{tabular}{|c|c|c|c|c|c|}
\hline Molecules & Observed & $\overline{\mathrm{Mod}}$ & $\overline{\mathrm{iA}}$ & Model B & References \\
\hline$\overline{{ }^{a} \mathrm{HCN}}$ & $6 \times 10^{-9}$ & $1.4 \times 10^{-9}$ & $2.4 \times 10^{-9}$ & $1.9 \times 10^{-9}$ & Irvine and Hjalmarson (1983) \\
\hline${ }^{a} \mathrm{NH}_{3}$ & $5 \times 10^{-8}$ & $4.5 \times 10^{-9}$ & $1.2 \times 10^{-9}$ & $3.9 \times 10^{-9}$ & Tölle et al. (1981) \\
\hline${ }^{a} \mathrm{CO}$ & $3 \times 10^{-5}$ & $1.8 \times 10^{-5}$ & $6.6 \times 10^{-5}$ & $2.6 \times 10^{-5}$ & Allen and Knapp (1978) \\
\hline${ }^{a} \mathrm{H}_{2} \mathrm{O}$ & $7 \times 10^{-8}$ & $4 \times 10^{-8}$ & $2.2 \times 10^{-8}$ & $1.6 \times 10^{-8}$ & Snell et al. (2000) \\
\hline${ }^{a} \mathrm{O}_{2}$ & $10^{-6}$ & $3.3 \times 10^{-7}$ & $1.9 \times 10^{-7}$ & $2.7 \times 10^{-7}$ & Gold et al. (2000) \\
\hline${ }^{a} \mathrm{CH}_{3} \mathrm{OH}$ & $5 \times 10^{-10}$ & $2.5 \times 10^{-11}$ & $4.4 \times 10^{-11}$ & $1.6 \times 10^{-11}$ & Friberg et al. (1988) \\
\hline${ }^{a} \mathrm{CH}_{3} \mathrm{CHO}$ & $3 \times 10^{-10}$ & $1.3 \times 10^{-13}$ & $9.1 \times 10^{-13}$ & $1.9 \times 10^{-13}$ & Matthews et al. (1985) \\
\hline${ }^{b} \mathrm{C}_{2} \mathrm{H}_{5} \mathrm{OH}$ & $1.5 \times 10^{-9}$ & $5.8 \times 10^{-17}$ & $3.6 \times 10^{-16}$ & $1.1 \times 10^{-15}$ & Ohishi et al. (1995) \\
\hline Alanine & - & $2.3 \times 10^{-17}$ & $8.3 \times 10^{-17}$ & $6 \times 10^{-17}$ & - \\
\hline Glycine & $\begin{array}{l}10^{-10 c} \\
7 \times 10^{-9 d} \\
(0.21-1.5) \times 10^{-9}\end{array}$ & $1.7 \times 10^{-14}$ & $2.9 \times 10^{-14}$ & $1.7 \times 10^{-14}$ & $\begin{array}{l}\text { Ceccarelli et al. (2000) } \\
\text { and } \\
\text { Kuan et al. (2003) }\end{array}$ \\
\hline
\end{tabular}

$\overline{\bar{a} \text { Observed near TMC-1 (10K) }}$

${ }^{b}$ Observed near Orion KL $(70 \mathrm{~K})$

${ }^{c}$ upper limit (cold cloud) ; ${ }^{d}$ upper limit (hot core)

freeze-out, the mass fractions in the innermost four shells start to decrease after reaching the saturation while the other shells do not decrease within the evolution time of the cloud. The evolution of the global average follows initially the evolution of the 10th shell, but afterwards, from around $t=2 \times 10^{13} \mathrm{~s}$, the deviation occurs as the global average is dominated by denser intermediate shells.

In Table 1, we present the results of Model A with Sets 1 and 2 chemical compositions, and the result from Model B using Set 1 composition (i.e., Model B1). We also compare the observed abundances of some of the species. For comparison, we converted mass fraction of a species into ratio of the number density of that species and that of $H_{2}$ so that our results are directly comparable with observables. The references from where the observed abundances have been obtained are provided in Column 6 .

From the Table, it is clear that our results in Sets 1-2 are generally close to the observational results for most of the lighter species. Since all the $\mathrm{C}$ and $\mathrm{O}$ are primarily channeled into the formation of $\mathrm{CO}$, production of other and more complex hydrocarbons by our method is not very efficient. For instance, for $\mathrm{C}_{2} \mathrm{H}_{5} \mathrm{OH}$, glycine, alanine, 
abundances obtained by our simulations are very low compared to the observed or 'upper limits' obtained from observations. According to Ohishi et al. (1995), $\mathrm{C}_{2} \mathrm{H}_{5} \mathrm{OH}$ is difficult to form in the gas phase. This is thought to be synthesized on the grain surfaces and then evaporated. Thus we believe that there could be other pathways than the method adopted by us to form glycine. These may be some of the reasons of the discrepancy seen in this Table.

\section{Concluding remarks}

In this paper, we presented the preliminary results on the chemical evolution inside a collapsing interstellar cloud. Our models are distinctly different from the other models used by several authors (Aikawa et al., 2005; Ceccarelli et al., 1996; Lim et al., 1999; Shalabiea and Greenberg, 1995; Shematovich et al., 1997). We do not include the heating and cooling processes while determining the dynamics of the cloud, and thus our model is not totally self-consistent. While in the outer edge of a diffused cloud the heating and cooling time scales are comparable to the infall time scales and should have been included, deep inside, the infall time scale is much shorter and cooling can be ignored. On the other hand, the outermost shell is also of very low density and thus the heating is low. The cooling is also negligible as the reactions rates are low. Thus we believe that even if the heating and cooling were included, the result would not have differed significantly.

Unlike the previous study Chakrabarti and Chakrabarti (2000a, 2000b) we have incorporated the grain chemistry of $\mathrm{H}_{2}$ formation self-consistently. This major improvement gave the most realistic abundances of $H_{2}$ molecules in the grain and the gas phases. We find that our computed average abundances generally agree with the observed abun- 
dances (see, e.g., Allen and Knapp, 1978; Friberg et al., 1988; Irvine and Hjalmarson, 1983; Matthews et al., 1985; Ohishi et al., 1995; Tölle et al., 1981) except when the molecules are complexes. We always seem to underestimate them as compared to the observed values or 'upper limits'. It is not clear at this moment how close the results of the bio-molecules are in comparison to the actual values, since the reaction rates we used are similar to the neutral-neutral rates which need not be accurate. If, for instance, the reaction rate at each step of glycine formation were higher by a factor of ten, the resulting glycine abundance would have been $10^{3}$ times higher, which is closer to observed claims. Given that the pathways to produce glycine may itself be different, perhaps one needs to look into laboratory experiments which simulate interstellar clouds for guidance (see, e.g., Elsila, 2007).

In future, we plan to improve the hydrodynamic model by including the angular motion and shock formation in the flow. We expect that jets and outflows would form and a part of this will fall back on the disk and the matter would be recycled. We also plan to improve the grain chemistry to include the formation of $\mathrm{CH}_{3} \mathrm{OH}, \mathrm{CO}, \mathrm{NH}_{3}, \mathrm{OH}$ on the grains themselves. Thus we anticipate that the chemical abundance will be strongly affected by such recycling of matter and such incorporation of newer species on the grain surfaces.

A. Das acknowledge the support from an ISRO project. 


\section{APPENDIX}

\section{SOLUTION PROCEDURE}

The equations are solved on a spherical grid extending from $r_{\text {in }}$ to $r_{\text {out }}$ composed of $N$ equal logarithmically spaced grids along the radial direction. The code is customized to take care of the collapsing spherical hydrodynamical flow which is strictly one dimensional. That is, no back flow is possible. Thus, to solve Eqs. (1-2) we use the first order upwind differencing method. After appropriately splitting, the Eqs. (1-2) become,

$$
\begin{aligned}
\rho_{i}^{j+1}= & \rho_{i}^{j}-\frac{d t}{r_{i}^{2}\left(r_{i+1}-r_{i}\right)}\left(\rho_{i+1}^{j} v_{r_{i+1}^{j}}^{j} r_{i+1}^{2}-\rho_{i}^{j} v_{r_{i}}^{j} r_{i}^{2}\right), \\
& \rho_{i}^{j+1} v_{r i}^{j+1}=\rho_{i}^{j} v_{r i}^{j}-\frac{d t}{r_{i}^{2}\left(r_{i+1}-r_{i}\right)} \\
& \left(\rho_{i+1}^{j}{ }^{2} v_{r i+1}^{j}{ }^{2} r_{i+1}^{2} / \rho_{i+1}^{j}-\rho_{i}^{j^{2}} v_{r i}^{j} r_{i}^{2} / \rho_{i}^{j}\right) \\
- & \frac{d t}{\left(r_{i+1}-r_{i}\right)}\left[\rho_{i}^{j}\left(\phi_{i+1}^{j}-\phi_{i}^{j}\right)+\left(p_{i+1}^{j}-p_{i}^{j}\right)\right]
\end{aligned}
$$

Here, $i$ denotes the index for the radial grid and $j$ denotes the index for the time. To avoid the instability in the code we chose the time step by using the Courant-Friedrichs-Lewy stability criterion, which gives,

$$
|v| \Delta t / \Delta r \leq 1
$$

i.e.,

$$
\Delta t \sim \Delta r /|v|
$$

where, $|v|$ is the magnitude of velocity, $\Delta t$ is the time step, and $\Delta r$ is the grid spacing along 
the radial direction. We always advance the time step after ensuring that the Courant condition is satisfied. To be on the safer side, we chose time step $d t=\Delta t / 2$. Even though we use self-gravitating flow, we do not solve Poisson equation to get the potential $\phi_{i}(r)$ here, since we are dealing with a spherical flow. Potential at any point is computed as a sum of two terms, one coming from the cloud itself $\left[\phi_{\text {cloud }}=-G M_{\text {cloud }}(r) / r\right.$, where, $M_{\text {cloud }}(r)$ is the mass of the cloud $r_{\text {in }}<r<r_{\text {out }}$ within the grid] and the other is due to the cloud core $\phi_{\text {core }}=-G M_{\text {core }} / r$, where, $M_{\text {core }}$ is the mass of the core within $r<r_{i n}$, the inner edge of the computational grid. The value of $\phi(r)$ that is required during the simulation is dynamically calculated from the mass within $r$, i.e., $\phi=-G\left(M_{\text {cloud }}+\right.$ $\left.M_{\text {core }}\right) / r$. Here, $M_{\text {cloud }}$ at $j$ th time step is calculated by adding contributions from each spherical shell,

$$
M_{\text {cloud }}^{j}=\Sigma_{i} 4 \pi r_{i}^{2} d r_{i} \rho_{i}^{j}
$$

where, $i=1,2, \ldots . . N$ and the densities at the $j$ th time step are used. We put $r=r_{\text {out }}$ to get the potential at the outer boundary.

\section{REFERENCES}

1. Acharyya, K., Chakrabarti, S. K., Chakrabarti, S., 2005. MNRAS 361, 550

2. Aikawa, Y., Herbst, E., Roberts, H., Caselli, P., 2005. ApJ 620, 330

3. Allen, M., Knapp, G. R., 1978. ApJ 225, 843

4. Bernstein, M. P., Dworkin, J. P., Stanford, S. A., Cooper, G. W., Mandela, L. J., 2002. Nature 416,401

5. Biham, O., Furman, I., Pirronello, V., Vidali, G., 2001. ApJ 553, 595 
6. Ceccarelli, C., Hollenbach, D. J., Tielens, A. G. G. M., 1996. ApJ 471, 400

7. Ceccarelli, C., Loinard, L., Castets, A., Faure, A., Lefloch, B., 2000. A\&A 362, 1122

8. Chakrabarti, S., Chakrabarti, S. K., 2000a. A\&A 354L, 6

9. Chakrabarti, S. K., Chakrabarti, S., 2000b. InJPB 74, 97

10. Elsila, J. E., 2007. ApJ 660, 911

11. Friberg, P., Madden, S. C., Hjalmarson A., Irvine, W. M., 1988. A\&A 195,281

12. Goldsmith, P. F., Melnick, G. J., Bergin, E. A. et al., 2000. ApJ 539L, 123

13. Gould, R. J., Salpeter, E. E., 1963. ApJ 138, 393

14. Hasegawa, T. I., Herbst, E., 1993. MNRAS 261, 83

15. Hasegawa, T. I., Herbst, E., Leung, C. M., 1992. ApJS 82, 167

16. Hollenbach, D., Salpeter, E. E., 1971. ApJ 163, 155

17. Hollenbach, D., Werner, M.W., Salpeter, E.E., 1971. ApJ 163, 165

18. Hollis, J. M., et al., 2003. ApJ 588, 353

19. Irvine, W. M., Hjalmarson, A., 1983. In Cosmo chemistry and the Origin of Life 113

20. Kuan, Y. J., Charnley, S. B., Huang, H. C., Tseng, W. L., Kisiel, Z., 2003. ApJ 593, 848

21. Leung, C. M., Herbst, E., Huebner, W. F., 1984. ApJS 56, 231

22. Lim, A. J, Rawlings, J. M. C., Williams, D. A., 1999. MNRAS 308, 1126

23. Matthews, H. E., Friberg, P., Irvine, W. M., 1985. ApJ 290, 609

24. Millar, T. J., Farquhar, P. R. A., Willacy, K., 1997. A\&A Suppl. Ser. 121139

25. Munoz Caro, G. M. et al., 2002. Nature 416, 403

26. Ohishi, M., Ishikawa, S. I., Yamamoto, S., Saito, S., Amano, T., 1995. ApJ 446L, 430

27. Prasad, S. S., Heere K. P., Tarafdar, S. P., 1991. ApJ 373, 123 
28. Prasad, S. S., Huntress, W. T., 1980a. ApJS 43, 1

29. Prasad, S. S., Huntress, W. T., 1980b. ApJ 239, 151

30. Shalabiea, O.M., Greenberg, J.M., 1995. A\&A 303, 233

31. Shematovich, V. I., Shustov, B. M., Wiebe, D. S., 1997. MNRAS 292, 601

32. Shu, F., 1977. ApJ 214, 488

33. Snell, R. L., et al., 2000. ApJ 539L, 101

34. Snyder L. E., et al., 2005. ApJ 619, 914

35. Sorrell, W. H., 2001. ApJ 555L 129

36. Tarafdar, S. P., Prasad, S. S., Huntress, W. T., Villere, K. R., Black, D. C., 1985. ApJ 289, 220

37. Tielens, A. G. G. M., 2005. The Physics and Chemistry of Interstellar Medium (Cambridge Univ. Press:Cambridge)

38. Tölle, F., Ungerechts, H., Walmsley, C. M., Winnewissen, G., Churchwell, E., 1981. A\&A 95, 143

39. Weingartner, J. C., Draine, B. T., 2001a. ApJS 134, 263

40. Weingartner, J. C., Draine, B. T., 2001b. ApJ 548, 296 